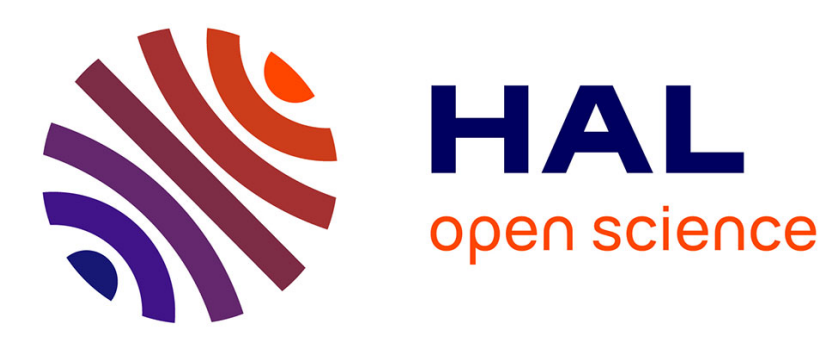

\title{
Septième partie. - Amorphes ioniques : caractères spécifiques d'une étude Mössbauer (isotope 57Fe)
}

\author{
F. Varret, M. Henry
}

\section{To cite this version:}

F. Varret, M. Henry. Septième partie. - Amorphes ioniques: caractères spécifiques d'une étude Mössbauer (isotope 57Fe). Revue de Physique Appliquée, 1980, 15 (6), pp.1057-1061. 10.1051/rphysap:019800015060105700 . jpa-00244823

HAL Id: jpa-00244823

https://hal.science/jpa-00244823

Submitted on 1 Jan 1980

HAL is a multi-disciplinary open access archive for the deposit and dissemination of scientific research documents, whether they are published or not. The documents may come from teaching and research institutions in France or abroad, or from public or private research centers.
L'archive ouverte pluridisciplinaire HAL, est destinée au dépôt et à la diffusion de documents scientifiques de niveau recherche, publiés ou non, émanant des établissements d'enseignement et de recherche français ou étrangers, des laboratoires publics ou privés. 


\title{
Septième partie. - Amorphes ioniques : caractères spécifiques d'une étude Mössbauer (isotope ${ }^{57} \mathbf{F e}$ )
}

\author{
F. Varret et M. Henry \\ Université du Maine, route de Laval, 72017 Le Mans Cedex, France
}

Parmi les amorphes ioniques, la spectrométrie Mössbauer s'est d'abord intéressée, de manière pratique, aux verres, puis par la suite aux composés définis. Ces derniers sont a priori plus simples et donc mieux adaptés à l'étude des phénomènes fondamentaux, structuraux et magnétiques, caractéristiques de l'état amorphe; c'est donc eux qui, dans la mesure du possible, nous serviront d'exemples.

Il y a un petit nombre de composés amorphes de composition définie étudiés par Mössbauer : $\mathrm{Fe}_{2} \mathrm{O}_{3}[1]$, $\mathrm{Fe}(\mathrm{OH})_{3}$ hydraté [2], le grenat GIG [3], $\mathrm{FeF}_{2}, \mathrm{FeCl}_{2}$, $\mathrm{FeBr}_{2}$ [4], $\mathrm{FeF}_{3}$ [5], $\mathrm{NaFeF}_{4}$ et $\mathrm{KFeF}_{4}$ [6]. Notre propos se limite donc à la spectrométrie de ${ }^{57} \mathrm{Fe}$.

L'apport spécifique de la spectrométrie Mössbauer peut provenir de quatre types de données expérimentales (que nous développerons plus loin):

a) La valeur du déplacement isomérique, donnant des informations sur la coordinence de l'ion Mössbauer.

b) Les distributions d'interactions quadrupolaires, plus aisément observables et interprétables dans les isolants que dans les amorphes métalliques, et devant déboucker sur des propriétés structurales.

c) Le profil du spectre à la limite des basses températures en présence d'un champ magnétique très élevé, permettant certaine connaissance de la structure magnétiquement gelée.

d) L'apparition progressive de l'interaction hyperfine et sa variation thermique, devant déboucher sur les propriétés de dynamique des spins.

Ces données prennent tout leur sens quand on les compare à celles des composés cristallisés correspondants; dans certains cas cette comparaison permet de caractériser l'état amorphe du composé (bien sûr l'usage d'autres techniques d'études est une précaution élémentaire !).

1. Coordinence de l'ion Mössbauer. - Le premier exemple d'une étude de coordinence est celui des ions $\mathrm{Fe}^{2+} \mathrm{Fe}^{3+}$ dans des verres oxydes alumino-silicates [7] : on distingue pour le Fer la coordinence 4 de la coordinence 6 par un abaissement d'environ $0,1 \mathrm{~mm}$ $\mathrm{s}^{-1} \mathrm{du}$ déplacement isomérique, par analogie avec de nombreux oxydes cristallisés.

Les déplacements isomériques des oxydes amorphes de composition définie déjà cités $\left(\mathrm{Fe}_{2} \mathrm{O}_{3}\right.$, $\mathrm{Fe}(\mathrm{OH})_{3}$ hydraté) correspondent tous à ceux des composés cristallisés correspondants; l'environnement de $\mathrm{Fe}^{3+}$ est donc le même, c'est-à-dire octaédrique (rien n'empêche de penser qu'on pourrait conserver dans l'état amorphe l'environnement tétraédrique réalisé dans un oxyde cristallisé).

Pour les fluorures, la coordinence 4 du Fer n'est pas a priori envisageable pour des raisons stériques; le problème ne se pose donc pas; les déplacements isomériques se correspondent parfaitement entre composés amorphes et cristallisés, et sans ambiguité l'environnement du fer est octaédrique.

2. Distributions d'interactions quadripolaires. - Le spectre paramagnétique se compose très généralement d'un doublet à raies larges (Fig. 1).

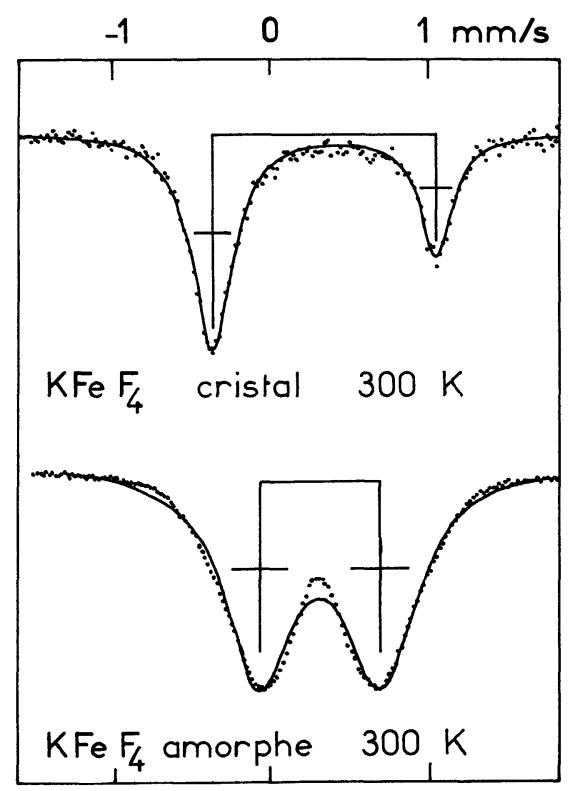

Fig. 1. - Fluorure $\mathrm{KFeF}_{4}$ sous forme cristallisée et amorphe (ajustement par des lorentziennes); on remarquera le caractère large et non lorentzien des raies du composé amorphe (d'après [6b]).

[Mössbauer spectra of $\mathrm{KFeF}_{4}$ in both amorphous and crystallized structures. A Lorentzian fit is not valid for the amorphous compound $[6 b]$.]

Il est intéressant de procéder à une analyse en distribution d'interactions quadripolaires, notée $P(S Q)$ dont quelques exemples sont indiqués sur la figure 2.

L'interprétation des données quadripolaires en termes de structure amorphe est un but ambitieux, mais pas inaccessible. En effet, les méthodes de calcul 

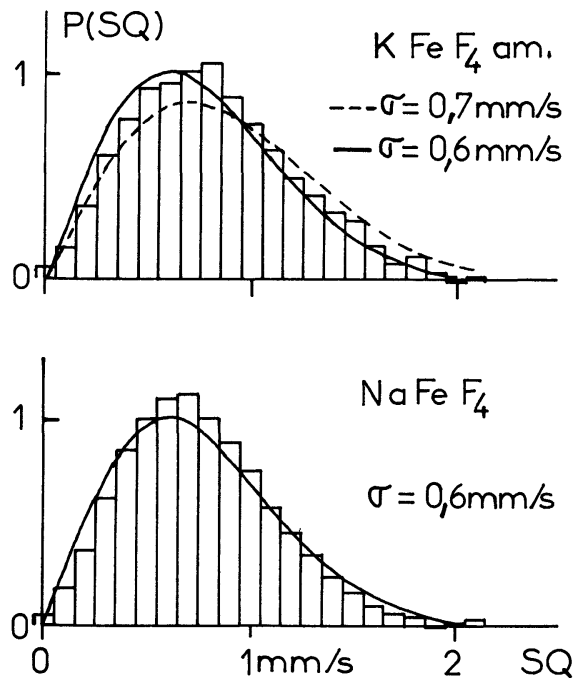

Fig. 2. - Analyse en distribution de séparations quadrupolaires ([6b]), les courbes tracées correspondent au modèle gaussien décrit dans le texte.

[Quadrupole splitting distribution in a Gaussian model as described in the text $[6 b]$.]

du gradient de $\mathrm{Fe}^{3+}$ dans les isolants cristallisés, par sommations sur le réseau (monopoles + dipôles [8]) donnent maintenant d'excellents résultats [9]; on peut donc espérer procéder de même avec les amorphes, et utiliser les données quadripolaires pour tester des modèles structuraux de l'empilement désordonné, lorsque c'est l'ion $\mathrm{Fe}^{3+}$ qui est concerné.

Jusqu'à présent, la méthode se limite à l'usage d'un modèle empirique de distribution d'interactions quadrupolaires aléatoires (analogue au modèle aléatoire de champ cristallin dans les métaux amorphes, voir par exemple [10]); ce modèle aboutit à une distribution de séparations quadrupolaires et de paramètres d'asymétrie. Un premier problème réside dans l'existence d'un signe de gradient de champ électrique : en effet, s'il y a autant de gradients positifs que de gradients négatifs, la recherche du signe n'a pas de sens, et le spectre de poudre en présence d'un champ magnétique intense reste symétrique (jusqu'à présent, seul $\mathrm{FeF}_{3}$ a été étudié en champ intense [11], et l'expérience à $300 \mathrm{~K}, 2 \mathrm{~T}$, n'a pas mis en évidence de dissymétrie mesurable du spectre). Malgré ce résultat négatif, on peut penser que la forme de la distribution de paramètre d'asymétrie se manifeste dans le profil du spectre en champ intense, et donc que les spectres en champ et hors champ ne donnent pas des informations redondantes.

Nous décrivons maintenant brièvement le modèle aléatoire imaginé par J. M. D. Coey [13], basé sur la remarque que l'on ne peut envisager, en vertu de l'équation de Poisson, trois distributions indépendantes des composantes principales du gradient de champ électrique : il faut se limiter à un plan (111, contenant l'origine) dans l'espace de coordonnées $V_{x x} V_{y y} V_{z z}$. Dans ce plan, on suppose par symétrie que la densité de probabilité ne dépend que de la distance $r$ à l'origine (Fig. 3).

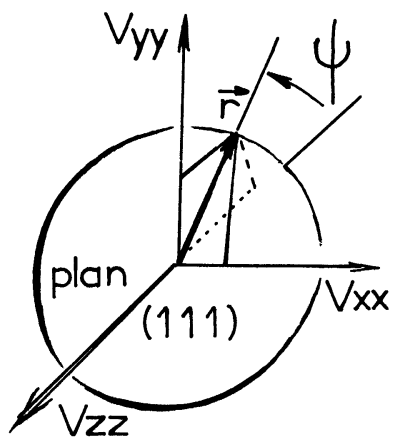

Fig. 3. - Espace de coordonnées $V_{x x} V_{y y} V_{z z}$; à chaque point du plan (111) passant par l'origine correspond un tenseur gradient. Une densité de probabilité $p(r)$ dans ce plan traite de manière symétrique les axes principaux $x y z$ (d'après J. M. D. Coey [13]).

[In $V_{x x} V_{y y} V_{z z}$ coordinates, each point of the (111) plan containing the origin corresponds to a field gradient tensor. For a probability density $p(r)$ in this plan the principal axes $x y z$ are equivalent [13].]

\section{Le calcul donne aisément :}

$$
\begin{gathered}
S Q=e Q V_{z z} \sqrt{1+\eta^{2} / 3}= \\
=e Q \sqrt{\frac{2}{3}\left(V_{x x}^{2}+V_{y y}^{2}+V_{z z}^{2}\right)}=e Q r \sqrt{\frac{2}{3}} \\
\eta=\sqrt{3} \operatorname{tg} \psi .
\end{gathered}
$$

Si l'on admet que la densité de probabilité est indépendante de l'angle $\psi$, on trouve immédiatement la distribution de paramètres d'asymétrie :

$$
P(\eta)=P(\psi) \frac{\mathrm{d} \psi}{\mathrm{d} \eta} \sim\left(1+\eta^{2} / 3\right) \text { dont l'allure est indi- }
$$

quée sur la figure 4. L'observation d'un tel type de distribution fournirait une excellente justification des hypothèses de symétrie du modèle; cette obser-

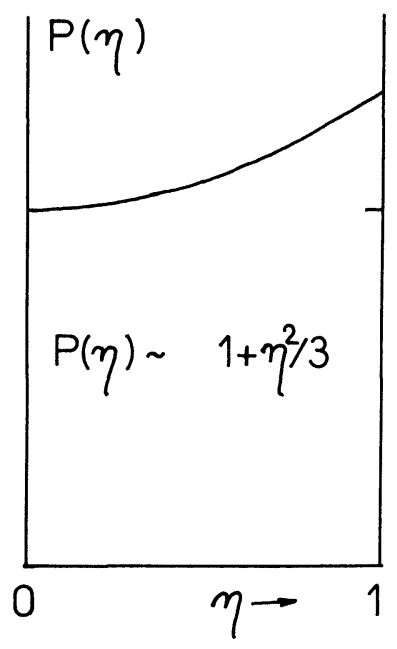

Fig. 4. - Distribution de paramètre d'asymétrie requise par la symétrie du modèle proposé (d'après J. M. D. Coey [13]).

[Distribution of asymmetry parameter consistent with the proposed model [13].] 
vation reste à faire, une des difficultés résidant dans la complexité des spectres sous champ appliqué.

La forme exacte de la distribution $P(S Q)$ dépend de la loi de densité de probabilité $p(r)$ dans le plan (111). $(p(r) r \mathrm{~d} r \mathrm{~d} \psi$ représente la proportion de noyaux ayant des composantes principales de gradient dans la surface élémentaire $r \mathrm{~d} r \mathrm{~d} \psi$ ). Supposons, par symétrie, que cette densité de probabilité soit donnée par une loi centrée sur l'origine, et de profil gaussien par simplicité :

$$
p(r) \sim \exp \left(-r^{2} / 2 r_{0}^{2}\right) .
$$

Il en résulte la loi : $P(S Q) \mathrm{d} S Q=p(r) \times 2 \pi r \mathrm{~d} r$

$$
P(S Q)=\frac{1}{\sigma^{2}} S Q \exp \left(-S Q^{2} / 2 \sigma^{2}\right) .
$$

Cette loi (normalisée) dépend d'un seul paramètre, et finalement se compare assez bien avec les résultats précis de $\mathrm{KFeF}_{4}$ et $\mathrm{NaFeF}_{4}$ (Fig. 2); pour l'instant il serait imprudent de conclure à la validité et surtout à la généralité du modèle aléatoire proposé.

Une dernière question concernant l'interaction quadrupolaire est la comparaison avec des grains extrêmement fins; on peut penser que les effets de surface deviennent majoritaires lorsque la dimension des grains devient inférieure à $10 \times 10 \times 10$ mailles (alors $50 \%$ des mailles sont déjà à la surface), l'aspect des raies quadrupolaires étant finalement celui d'un amorphe. Il est alors nécessaire de recouper les résultats quadrupolaires avec d'autres données, chercher un éventuel défaut de reproductibilité des échantillons (et de leurs spectres) qui serait indicatif d'effets de taille de grains.

3. Etat magnétiquement gelé (limite des basses températures). - De manière générale, la distribution des champs hyperfins à saturation, dans le cas de composés ferriques (état $\mathbf{S}$ ) est faible, et la spectrométrie Mössbauer se prête particulièrement bien à l'étude de la structure magnétique.

Une description de diverses structures possibles a été donnée par J. M. D. Coey [14]. Jusqu'à présent, deux types de structure ont été observés :

a) La structure ferromagnétique, dans $\mathrm{FeF}_{2}$ [15], par mesures d'aimantation.

b) La structure speromagnétique, proposée par Coey pour expliquer la faible aimantation de $\mathrm{Fe}(\mathrm{OH})_{3}$ $0,9 \mathrm{H}_{2} \mathrm{O}$ [2] (les moments magnétiques sont orientés au hasard, de manière isotrope).

La spectrométrie Mössbauer sous champ intense est la technique adaptée à l'étude des divers types de structure magnétique (voir [16] par exemple); le calcul du spectre résultant ne présente pas de difficultés particulières [17]. Parmi les fluorures, $\mathrm{FeF}_{3}$ et $\mathrm{KFeF}_{4}$ ont été étudiés en champ intense [11,12] : les spectres obtenus (Fig. 5) ont des élargissements caractéristiques d'une structure speromagnétique

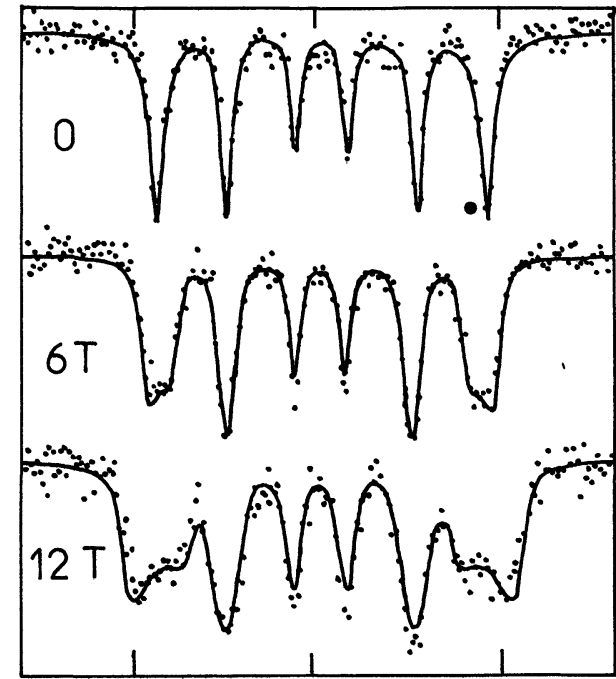

Fe $F_{3}$ amorphe $4,2 \mathrm{~K}$

Fig. 5. - Caractère speromagnétique de $\mathrm{FeF}_{3}$ amorphe $[11,12]$ : spectres en champ appliqué parallèle à la direction d'observation, à $4,2 \mathrm{~K}$.

[Speromagnetism in amorphous $\mathrm{FeF}_{3}$ [11], [12]. Mössbauer spectra with an external magnetic field applied parallel to the $\gamma$ rays at $4.2 \mathrm{~K}$.]

(d'autres structures ont été mises en évidence dans des alliages [18]).

Un phénomène très généralement observé avec les amorphes ferriques est la réduction du champ hyperfin à saturation par rapport aux composés cristallisés analogues de dimension magnétique 3 (Tableau I). On peut tout d'abord éliminer l'effet de la covalence, car le déplacement isomérique est, pour tous les amorphes cités, le même que dans l'état cristallisé. Il reste donc une explication analogue à celle des systèmes de basse dimensionnalité [19], celle du mouvement au point zéro; celui-ci toutefois nécessite la manifestation d'ondes de spin dans les matériaux amorphes concernés, et pour l'instant seuls les ferromagnétiques en ont donné l'évidence (voir par exemple [14] et [20]). La question reste donc ouverte.

Une autre observation générale réside dans l'absence de corrélation, pour les amorphes ferriques, entre l'interaction quadrupolaire et l'orientation du moment magnétique (celle-ci se manifesterait par une dissymétrie du spectre à basse température). Cette absence correspond sans doute à la faiblesse de l'anisotropie locale dans ces composés, la structure speromagnétique étant gouvernée uniquement par les interactions d'échange (ces dernières semblent donc peu corrélées aux interactions quadrupolaires...).

\section{Evolution thermique de l'interaction hyperfine} magnétique. - De manière caractéristique, les systèmes désordonnés présentent un passage très progressif de la température d'ordre magnétique; dans une large zone de température, on observe alors des spectres aux raies très élargies, dont le profil 
Tableau I. - Champ hyperfinà saturation de quelques composés ferriques $(\mathrm{kOe})$.

\begin{tabular}{|c|c|c|c|c|c|c|c|}
\hline & $\mathrm{Fe}_{2} \mathrm{O}_{3}$ & $\begin{array}{c}\text { GIG } \\
-\end{array}$ & $\begin{array}{c}\mathrm{Fe}(\mathrm{OH})_{3} \\
\text { (hydr.) } \\
-\end{array}$ & $\mathrm{FeF}_{3}$ & $\mathrm{NaFeF}_{4}$ & $\begin{array}{c}\mathrm{KFeF}_{4} \\
-\end{array}$ & $\begin{array}{c}\mathrm{K}_{2} \mathrm{FeF}_{5} \\
-\end{array}$ \\
\hline Cristallisé & 535 & 478 & - & 618 & 562 & 540 & 410 \\
\hline Amorphe & 470,460 & 460 & 460 & 560 & $\sim 530$ & $\sim 520$ & - \\
\hline [référence] & {$[1 a, b]$} & [3] & [2] & [5] & [6] & [6] & [19] \\
\hline$(\operatorname{dim})$ & (3) & (3) & (3) & (3) & (2) & (2) & (1) \\
\hline
\end{tabular}

(dim est la dimensionnalité magnétique du système cristallisé.)

peut s'interpréter indifféremment par une distribution statique de champs hyperfins, ou par une fréquence de fluctuations superparamagnétiques de grandeur comparable à la fréquence de Larmor du champ hyperfin.

Le cas des amorphes ioniques étudiés semble analogue à celui des "verres de spin proprement dits" (obtenus par dilution d'un ferromagnétique) : chaque spin se gèle sur place, la fréquence de ses fluctuations diminuant lorsque la température diminue : on obtient alors, pour chaque technique de mesure, une température de gel d'autant plus basse que la fréquence caractéristique de la mesure est plus basse (une discussion détaillée de cette propriété est donnée dans [21]). Des résultats concernant $\mathrm{FeF}_{3}$ amorphe sont indiqués sur la figure 6 ; on remarquera que le Mössbauer voit des spins gelés à température plus élevée que les mesures magnétiques. Par ailleurs, il semble exister une sorte de loi d'échelle reliant la fraction paramagnétique (Mössbauer) et la température, car en échelle réduite les données relatives aux trois fluorures $\mathrm{FeF}_{3}, \mathrm{KFeF}_{4}, \mathrm{NaFeF}_{4}$ sont remarquablement identiques (il est donc tentant de chercher un modèle simple ; toutefois le modèle de domaines superparamagnétiques, qui semble adéquat dans des verres magnétiquement dilués [22], est exclu pour nos fluorures à cause de leurs propriétés magnétiques [12]).

(L'interprétation précise de tous les spectres élargis semble un problème redoutable, car il se superpose des causes dynamiques et statiques d'élargissement, les premières étant censées prépondérantes au voisinage de la température de gel, les secondes à température intermédiaire.)

L'observation précise de la variation thermique du champ hyperfin à très basse température (disons jusqu'à $0,2 \mathrm{Tg}$ ), sera nécessaire avant de conclure à l'existence d'ondes de spin de grande longueur d'onde (décroissance du champ hyperfin en $\mathrm{T}^{3 / 2}$ ).

5. Amorphes et verres. - Cette description brève de l'apport de la spectrométrie Mössbauer s'est limitée aux phénomènes caractéristiques de l'état amorphe et aux isolants de composition définie; ces phénomènes caractéristiques peuvent bien sûr se manifester également dans la très grande diversité des verres ioniques d'origine ou de destination industrielles. Les problèmes structuraux des verres, et particulièrement les

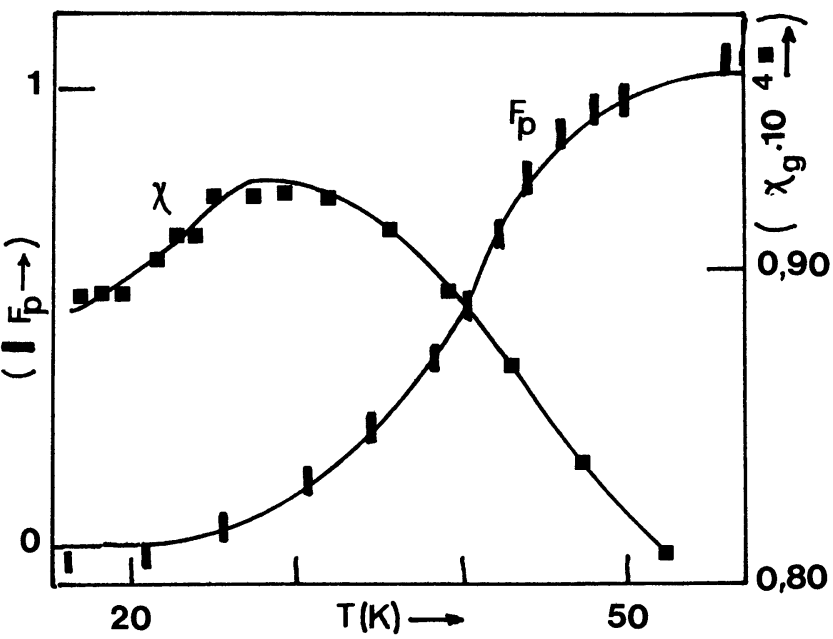

Fig. 6. - Fraction paramagnétique dans le spectre Mössbauer, et susceptibilité magnétique de $\mathrm{FeF}_{3}$ amorphe [5]; la température d'apparition de la thermorémanence $\left(T_{\mathrm{RM}}\right)$ est $29 \mathrm{~K}$.

[Paramagnetic fraction obtained from Mössbauer spectra and magnetic susceptibility in amorphous $\mathrm{FeF}_{3}$ [5]. The thermoremanence temperature $\left(T_{\mathrm{RM}}\right)$ is $29 \mathrm{~K}$.]

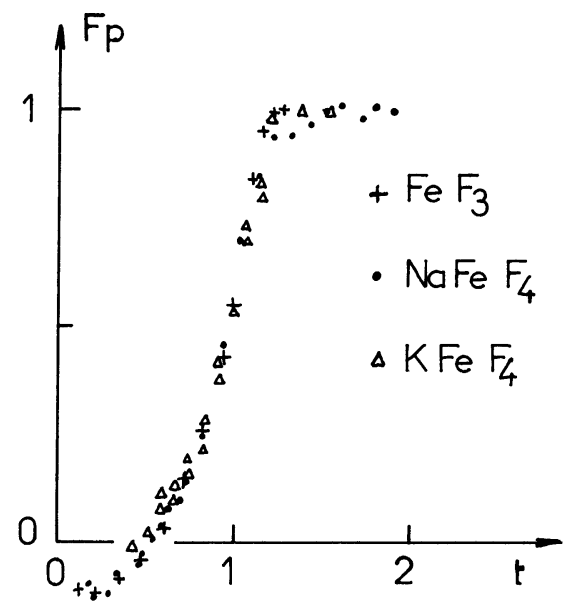

Fig. 7. - Fraction paramagnétique Mössbauer, pour $\mathrm{FeF}_{3}(+)$, $\mathrm{NaFeF}_{4}(),. \mathrm{KFeF}_{4}(\triangle)$; les échelles de température sont respectivement $(\mathrm{T} / 39),(\mathrm{T} / 20,5),(\mathrm{T} / 12,5)$.

[Paramagnetic fraction in $\mathrm{FeF}_{3}(+), \mathrm{NaFeF}_{4}($.$) and \mathrm{KFeF}_{4}(\Delta)$ vs. Temperature in respective scale $\mathrm{T} / 39, \mathrm{~T} / 20.5$ and $\mathrm{T} / 12.5$.]

problèmes de cristallisation font actuellement l'objet de recherches actives [23] qui mériteraient aussi une étude synthétique. 


\section{Bibliographie}

[1] Van Diepen, A. M., Popma, T. J. A., J. Physique Colloq. 37 (1976) C6-755 et Solid State Commun. 27 (1977) 121 ; Shigematsu, T., Bando, Y., Takada, T., J. Physique Colloq. 40 (1979) C2-153

[2] Coey, J. M. D., Readman, P. W., Nature 246 (1973) 476.

[3] Popma, T. J. A., Van Diepen, A. M., Mat. Res. Bull. 9 (1974) 1119.

Matsuzawa, T., Okamura, K., Shishido, T., Yajima, S., J. Physique Colloq. 40 (1979) C2-149.

[4] Litterst, F. J., Kalvius, G. M., Boyle, A. J. F., J. Physique Colloq. 35 (1974) C6-403.

Boyle, A. J., Kalvius, G. M., Gruen, D. M., Clifton, J. R., Mc Beth, R. L., J. Physique Colloq. 32 (1971) C1-224.

[5] Ferey, G., Leclerc, A. M., De Pape, R., Mariot, J. P., VARRET, F., Solid State Commun. 29 (1979) 477.

[6] Ferey, G., Coey, J. M. D., Henry, M., Varret, F., MasseNET, O., Int. Conf. Magnetism (Munich, Septembre 1979); Int Conf. Mössbauer Spectrometry (Portoroz, Septembre 1979).

[7] Kurkjian, C. R., Sigety, E. A., Phys. Chem. Gilasses 9 (1968) 73.

[8] Le gradient dans les $\mathrm{AFeF}_{4}$ cristallisés est dû principalement à la polarisation des fluors (Y. Calage, travail en cours).

[9] Voir par exemple Calage, Y., Thèse, Rennes, 1976.
[10] Fert, A., Campbell, I. A., J. Phys. F : Metal Phys. 8 (1978) L 57.

[11] Expérience réalisée au SNCI, Grenoble.

[12] Ferey, G., Varret, F., Coey, J. M. D., J. Physics C 13 (1979) 351.

[13] Coey, J. M. D., CzJzeK, G., calculs non publiés.

[14] Coey, J. M. D., J. Appl. Phys. 49 (1978) 1646-52.

[15] LitTerst, J., J. Physique Lett. 36 (1975) 197.

[16] Chappert, J., Teillet, J., Varret, F., Int. Conf. Solids and Plasmas in high magnetic fields (Boston, Septembre 1978).

[17] Teillet, J., Varret, F., Programmes de calcul non publiés.

[18] Coey, J. M. D., Chappert, J., Reboulllat, J. P., Wang, T. S., Phys. Rev. Lett. 36 (1976) L-1061;

Chappert, J., J. Physique Colloq. 40 (1979) C2-107.

[19] Gupta, G. P., Dickson, D. P. E., Johnson, C. E., J. Physique Colloq. 40 (1979) C2-304.

[20] Chien, C. L., Hasegawa, R., Amorphous Magnetism II, ed. R. A. Levy et R. Hasegawa (Plenum Press, New York) 1977, page 289.

[21] Murani, A. P., J. Magnetism and Magnetic Materials 5 (1977) 95.

[22] Bieman, L. H., Kenealy, P. F., de GraA, A. M., Amorphous Magnetism II (voir [20]) page 587

[23] Friedt, J. M., communication privée. 